NOTICE: this is the author's version of a work that was accepted for publication in Acta Materialia. Changes resulting from the publishing process, such as peer review, editing, corrections, structural formatting, and other quality control mechanisms may not be reflected in this document. Changes may have been made to this work since it was submitted for publication. A definitive version was subsequently published in Acta Materialia, 59, 17, 2011 DOI 10.1016/j.actamat.2011.06.018 


\title{
Damage evolution and rupture time prediction in thermal barrier coatings subjected to cyclic heating and cooling: an acoustic emission method
}

\author{
L. Yang ${ }^{[1,2]}$, Y. C. Zhou ${ }^{[1,2] *}$, C. $\mathrm{Lu}^{[3] *}$ \\ ${ }^{[1]}$ Key Laboratory of Low Dimensional Materials \& Application Technology (Ministry of \\ Education), Xiangtan University, Xiangtan, Hunan 411105, China \\ ${ }^{[2]}$ Faculty of Materials, Optoelectronic \& Physics, Xiangtan University, Xiangtan, Hunan \\ 411105, China \\ ${ }^{[3]}$ Department of Mechanical Engineering, Curtin University, Perth, Western Australia 6845, \\ Australia
}

\begin{abstract}
The real-time testing and quantitative assessment of damage evolution in thermal barrier coatings (TBCs) is desirable, but still intractable, especially at elevated temperature. In this paper, the fracture process of TBCs subjected to cyclic heating and cooling is monitored using an acoustic emission method. Based on the wavelet analysis of acoustic emission signals, damage modes in TBCs are discriminated. The results show that, due to thermal stress, it is preferential for vertical cracks in the heating stage and interface cracks in the cooling stage. The surface crack density and interface crack length are calculated to obtain the quantitative correlation of damage evolution in TBCs and acoustic emission parameters. The rupture time of TBCs can be predicted by the statistical analysis of acoustic emission signals.
\end{abstract}

Key words Thermal barrier coatings, acoustic emission, damage mode, damage evolution, rupture time prediction

\footnotetext{
*Corresponding authors: e-mail: zhouyc@xtu.edu.cn (Y.C. Zhou); c.lu@ curtin.edu.au (C. Lu).
} 


\section{Introduction}

Thermal barrier coatings (TBCs) such as $\mathrm{ZrO}_{2}-8 \mathrm{wt} . \% \mathrm{Y}_{2} \mathrm{O}_{3}$ have great potential for many applications in gas turbine, internal combustion engine and fuel evaporator due to their excellent thermal protection, high hardness and wear resistance [1-3]. They can separate and protect structural materials against hot gas temperature to improve engine efficiency and performance. As illustrated in Fig. 1, TBCs commonly comprise a ceramic top-coat that increases the operation temperature of turbine components, a substrate that endures mechanical loading, and a MCrAlY alloy (M represents $\mathrm{Ni}$, Co, or Fe) bond-coating that enhances adhesion of the ceramic coat to substrate. This typical kind of structure makes each layer of TBCs have remarkably different physical, thermal, and mechanical properties, which result in thermal stress in TBCs. During processing and further thermal exposure, a fourth layer, named thermally grown oxide (TGO), is formed between bond and top coats due to diffusion and reaction of oxygen and metal iron. The growth of TGO aggravates the mismatch between these layers. Moreover, TBCs usually withstand the most extreme temperature, temperature cycling, and stress conditions. The complex shape, structure, and harsh operating conditions make the failure behavior of TBCs much more complicated than that in any metallic, ceramic components or other coating systems, which show a poor service life in the way of unpredictable rupture time, position and types. Therefore, TBCs are regarded as one of the most complex coating systems [1].

Significant efforts have been dedicated towards reliable assessments of failure behavior of TBCs and improving their reliability. For instance, delamination is attributed to residual stress induced by the thermal mismatch and spatial variations in the growth of TGO [4-5]. Meanwhile, numerous theoretical and finite element calculations, based on the stress 
distribution, interface strength, morphological instability of TGO, have been carried out to qualitatively explain how cracks propagate [5-8]. However, the heterogeneous and complex microstructures of TBCs may result in numerous crack sites and failure modes with a superposition of different mechanisms, in which their reliability cannot be accurately assessed by only one failure mode. Furthermore, the failure behavior of TBCs is not only dependent on their own mechanical properties and constitutive relationships, but also on their surface and interface effects as well as mechanical properties of substrate. This makes the failure assessment of TBCs very difficult by using traditional strength theories or experimental mechanics methods. There is a growing interest in bringing together experiments under destructive load with ideas from in-situ or real time nondestructive tests [9-17]. The latter can provide viable and direct failure information, which is desirable and necessary for understanding failure mechanisms of TBCs.

A number of methods have been applied in the nondestructive testing and lifetime prediction of TBCs, such as X-ray diffraction [9], impedance spectroscopy [10], infrared spectroscopy [11], $\mathrm{Cr}^{3+}$ piezospectroscopy [12], synchrotron radiation [13], and acoustic emission (AE) [14-17]. Among these methods, AE has attained an ever-increasing concern because it can be used to continuously detect the intrinsic damage by microcracking in a material without strict testing conditions, which is very difficult with other methods. The fracture behavior of TBCs under mechanical or thermal loads such as tensile, bend, torsion, thermal shock and fatigue, has been qualitatively investigated by the duration, count, energy and amplitude of AE signals [14-18]. The combined effect of bond coat oxidation and mechanical load on fracture properties of TBCs has also been considered [17,18]. Generally, there are two main failure modes in TBCs, including surface vertical crack in ceramic coating 
and crack along the top/bond or TGO interface $[17,18]$. When subjected to external mechanical loading or temperature, these failures in TBCs are activated by stresses that arise because of the strain mismatch between coating and other layers, and meanwhile cause AE signals, as schematically illustrated in Fig. 1. The results of $\mathrm{AE}$ assessment revealed that coating damage commonly originates from vertical cracks and then follows by delamination under tensile or bend conditions $[17,18]$. However, there is lack of quantitative verification in all these studies. Several important problems on their lifetime prediction are still intractable, such as on how to discriminate cracking modes (e.g., vertical cracking, interface cracking), how to quantify the damage evolution, and how to predict the occurrence time of catastrophic failure in TBCs.

Our recent experiments have shown that the frequency spectra of AE signals are strongly dependent on the fracture types of TBCs [19], which was also revealed in large scale rupture such as volcano seismicity [20]. These findings indicate that the damage characteristic of TBCs can be obtained by signal processing methods such as wavelet transformation, which can demonstrate both time and frequency domains of AE signals [21-23]. As shown in Fig. 1, a key problem in the quantitative $\mathrm{AE}$ assessment is, thus, to find out the relationship between damage evolution parameters (e.g., crack density, crack length) and AE features. For a brittle film on a ductile substrate, McGuigan et al [24] developed an elastic-plastic shear lag model to analytically calculate the crack density under tensile loading. With a shear lag approximation, the model considers an elastic-plastic interlayer, in which the load transfer from thick ductile substrate to thin brittle film can be described. This agrees well with the structure of TBCs, indicating an opportunity for theoretical prediction on coating failure in TBCs. In their experiments, Choi et al [25] observed the delamination crack propagation in 
TBCs under laser thermal cyclic loading, but the real-time monitoring on thermal conductivity failed to give direct and quantitative information on damage degree, which is necessary for the real time testing of TBC failure.

While micro-cracks are vital to the TBC performance, their propagation and coalescence are not catastrophic until the large-scale cleavage or spallation of coating occurs. An important issue shown in Fig. 1 is how to predict the critical point, at which a cascade of events develops at increasingly large scales, culminating in a large spalling. Numerous theoretical life prediction models based on the mismatch strain and the thickness of oxide layer have been proposed, which might empirically predict the mechanical integrity of TBCs. Nevertheless, the complex and varied fracture behavior of TBCs is not just the results of mismatch properties and oxidation, which implies that these models are limited to accurately estimating the lifetime of TBCs. Busso et al [6] recently predicted their lifetimes based on a statistical treatment of morphological characteristics and non-destructive stress measurements of TBCs. Here, a key question regarding the potential reliability prediction is, therefore, whether it is possible to predict the precursory time of catastrophic coating failure by means of the statistical analysis on AE signals in a fracture process.

In this paper, the thermal fracture behavior of TBCs is investigated under cyclic heating and cooling tests. First, the AE response of TBC specimens during a full thermal cycling process is recorded and analyzed to characterize crack modes by wavelet transformation. Then, the surface vertical crack density and interface crack length are carefully assessed, based on the elastic-plastic shear lag model developed by McGuigan et al [24] and the analysis of interface intensity factors [25]. The results obtained from the assessment can be used to analyze the correlation of damage evolution in TBCs and AE characteristic. Finally, a 
statistical analysis of $\mathrm{AE}$ activities is conducted to predict the critical point or the precursory time of ultimate coating failure.

\section{Testing and analysis methodology}

\subsection{TBC specimens}

Super-alloy DZ125 was chosen as substrate and the $\mathrm{NiCr}_{22} \mathrm{Al}_{7} \mathrm{Y}_{0.2}$ (wt.\%) bond coating was deposited on one surface of substrate by the vacuum plasma-spray method with a Sulzer-Metco system. Then, a $\mathrm{ZrO}_{2}-8 \mathrm{wt} . \% \mathrm{Y}_{2} \mathrm{O}_{3}$ ceramic coat was prepared using electron beam physical vapor deposition. The thicknesses of substrate, bond coating and ceramic coat are $3 \mathrm{~mm}, 50 \mu \mathrm{m}$ and $100 \mu \mathrm{m}$, respectively. The length and width of TBC specimens are 40 $\mathrm{mm}$ and $5 \mathrm{~mm}$, respectively.

\subsection{AE monitoring}

The cyclic heating and cooling tests of TBC specimens were carried out in a resistance-heated furnace with temperature of $800^{\circ} \mathrm{C}$. A cycle was defined as a 5-minute heating in furnace and followed a 10-minute cooling in air at room temperature. Here, it is worth noting that the full process was performed in air or oxidizing atmosphere. The AE monitoring schematic of TBCs during cyclic heating and cooling is shown in Fig. 2. One end of a platinum wire was spot-welded onto the substrate surface, and the other end was attached firmly to an AE sensor through an elastic cord and butter couplant. The platinum wire can keep the AE sensor operating at room temperature and be freely moved with specimen. Therefore, AE signals occurred in both heating and cooling stages could be sampled. The sampling rate was set to $1 \mathrm{MHz}$ with a DB16 AE monitoring system, the amplification factor 
was 400, and the threshold was $30 \mathrm{mV}$. To avoid mechanical and electromagnetic noise, the low- and high-filter frequencies were set to $0.1 \mathrm{kHz}$ and $1 \mathrm{MHz}$, respectively.

\subsection{Analysis of AE signals}

$\mathrm{AE}$ is a non-stationary process, i.e., the power spectrum changes with time, where shortand long-time phenomena coexist. For this reason, a scale-time procedure such as wavelet transform is an ideal tool to manage data. Wavelet transform is not a single set of basis functions like the Fourier transform, which utilizes just sine and cosine functions. Instead, wavelet transform has a very large set of possible basis functions [21-23]. Thus, wavelet analysis provides an immediate access to information that can be obscured by time-frequency methods. The idea of wavelet analysis is to slice data into a set of wavelet components, which are called wavelet levels. Each wavelet level has its specific frequency range. For a given AE signal, $f(t)$ can be written as a summation of a series of decomposed sub-signals, that is

$$
f(t)=f^{0}(t)+f^{1}(t)+\cdots f^{n}(t)
$$

where $f^{0}(t), f^{1}(t), \cdots f^{n}(t)$ are the levels of decomposed signals, which are measured by their energy contents. The energy content in each level can be calculated by using $E^{(j)}(t)=\sum_{\tau=t_{0}}^{t}\left(f^{(j)}(\tau)\right)^{2}$ and then the total energy is given by $E^{(T)}(t)=\sum_{j=0}^{n} E^{(j)}(t)$. The energy components in these levels are different and are closely relevant to failure modes. Thus, the energy coefficient, defined as the ratio of energy in different levels to the total energy, i.e., $R^{(i)}(t)=E^{(i)}(t) / E^{(T)}(t)$, is useful to distinguish different AE signals from various possible modes in TBCs. A more detailed discussion on wavelet transform is given in Refs. [19, 21-23]. 
Based on wavelet transform, the energy coefficient $R^{(i)}(t)$ was calculated by using Matlab programs with 'db8' (a member of the Daubechies wavelets family) wavelet. The analyzing scale $n$ was restricted to 5, and AE signals were decomposed into 6 levels with the particular frequency ranges of D1 (0.25-0.5 MHz), D2 (0.125-0.25 MHz), .., C5 (0-156.25 Hz). Two typical distribution histograms of energy coefficients, as shown in Fig. 3 are found and their maximum energy coefficients are concentrated on levels D1 and D2, respectively. Obviously, they are representative frequency bands of surface vertical cracking in ceramic coat or interface cracking between ceramic and bond coating, which are two main failure modes in TBCs. In our recent work on the tensile failure process, the representative frequency bands of surface cracking and interface cracking have been quantified with their maximum energy coefficients on levels D1 and D2, respectively, which is the same as shown in Fig. 3 [19]. Therefore, the representative frequency bands of failure modes in TBCs under thermal cycling can be also quantified. This result reveals that the frequency spectra from AE signals are strongly dependent on failure modes of materials, but they are almost independent of the failure size and operating pressure [19, 20, 26].

\section{Damage evolution in TBCs}

\subsection{AE and coatings failure characteristics}

Figure 4 shows the AE events in TBC specimens during cyclic heating and cooling tests. Two different regions with respect to the number of AE events can be distinguished: (1) AE events increase slowly during initial 110 thermal cycles, and (2) AE events increase rapidly with the increase of cycle number. To characterize failure in coatings, the microstructure development subjected to cyclic heating and cooling was observed by using scanning electron 
microscope. Moreover, two specimens were employed and their corresponding cross-sectional patterns at various cycles are shown in Fig. 5. Small microcracks appear in ceramic coating at the early stage of, for example, 20 cycles. These microcracks propagate and merge with each other to form surface vertical cracks that can be seen after 60 cycles. Then, surface vertical cracks propagate and meanwhile interface cracks occur in the ceramic/bond coating, which increase in both length and number with the increase of cycle number. An abrupt change in Fig. 4 is referred to as a large energy release, implying an incurrence of serious damage in TBCs, however, no visible coating spallation appears in the cross-sections of TBCs, as shown in Fig. 5. These results imply that the trend of the total AE events is not enough to assess the failure of TBCs, such as, the occurrence time of interface cracks. It is also seen that failures are not uniformly distributed in a chosen cycle for two different samples. Here, the non-uniformity is mainly attributed to the inherent heterogeneity and incomplete observation of TBC sections, which indicates that it is difficult to study the damage evolution and correlation with $\mathrm{AE}$ features just by observed microstructures.

To analyze the damage behavior of TBCs, AE events and their corresponding amplitudes during heating and cooling stages were measured. As shown in Fig. 6, AE signals were motivated in both cooling and heating stages, whereas the latter was commonly regarded as unimportant to TBCs. The AE amplitudes are mainly distributed in a range of 30 to $500 \mathrm{mV}$. Although there is a little increase of $\mathrm{AE}$ amplitudes with increasing the cycle number, no noticeable regions were found with respect to amplitudes. Moreover, AE amplitudes in the heating stage show the same range and irregular distribution as those in the cooling stage, indicating that $\mathrm{AE}$ signals monitored in the heating stage are not noise. These results demonstrate that micro-failures of TBCs occur in both cooling and heating stages. As we 
know, $\mathrm{AE}$ activities of TBCs are a mixture result of coating failures and other factors, such as substrate deformation and noise. Consequently, the comparison of AE amplitudes between heating and cooling stages is unreliable to discriminate the mode of coating failure, which is the key to understand failure mechanisms of TBCs.

In terms of wavelet transform in Section 2.3, the failure mode differentiation of $\mathrm{AE}$ signals can be realized and sampled during heating and cooling stages. As shown in Fig. 7, AE signals are classified into three classes; one for surface vertical cracking with the maximum energy coefficient at level D1, one for interface cracking with the maximum energy coefficient at level D2, and another for others such as substrate deformation and noise. Failures occurred in the heating stage are mainly surface vertical cracking but those occurred in the cooling stage exhibit interface cracking. The surface vertical cracking appears at the beginning of cycle tests and keeps actively and gradually increasing during the whole cycle. The surface vertical cracking occurs more frequently than interface cracking during the initial cycles. The interface cracking occurs until cycles up to 40 and abruptly increases at 110 cycles. These results imply that cracking in ceramic coating is easier to form than that interface for TBCs subjected to thermal cycles. However, such a tendency becomes inversely with an abrupt increase of interface cracking during the latter cycles.

\subsection{Failure mechanism of TBCs}

Surface vertical or interface cracks are strongly dependent on the transient thermal load in TBCs. To correlate the monitored AE signals with internal damage, stresses in TBCs subjected to cycle heating and cooling were analyzed. Generally, stresses in TBCs are composed of thermal stress, induced by the mismatch of thermal expansion coefficients 
between substrate and coatings, and initial residual stress, attributed to rapid contraction of sprayed splat and cooling from deposition temperature. Residual stress in coating from a preparation process is compressive because of the small thermal expansion coefficient of ceramic compared to that of alloy substrate. Numerous experimental and theoretical results revealed that the compressive residual stress is commonly in the range of -40 to $-100 \mathrm{MPa}$ [27-30]. If bond coating is simplified to the same layer as substrate and TBCs is only subjected to elastic deformation, thermal stress in TBCs, based on the strain compatibility at interfaces, can be expressed as [31,32],

$$
\sigma=\frac{E_{t}^{*}\left[E_{s}^{*} h_{s}\left(\alpha_{s}-\alpha_{t}\right)\left(T-T_{r}\right)\right]}{E_{s}^{*} h_{s}+E_{t}^{*} h_{t}}+\sigma_{r}
$$

and thus, thermal strain in top coating is,

$$
\varepsilon=\frac{E_{s}^{*} h_{s}\left(\alpha_{s}-\alpha_{t}\right)\left(T-T_{r}\right)}{E_{s}^{*} h_{s}+E_{t}^{*} h_{t}}+\frac{\sigma_{r}}{E_{t}^{*}}
$$

where $E_{i}^{*}=\frac{E_{i}}{1-v_{i}}(i=t$ or $s), E, v, \alpha$ and $h$ are Young's modulus, Poisson's ratio, thermal expansion coefficient, and thickness, respectively. The subscripts $t$ and $s$ represent top coating and substrate. The values of $E, v$, and $\alpha$ for ceramic coating are $48 \mathrm{GPa}, 0.1$, and $9 \times 10^{-6}$ and their values for bond coating are $200 \mathrm{GPa}, 0.3$, and $14.8 \times 10^{-6}$, respectively [27-30]. $T$ is temperature, $T_{r}$ is room temperature, and $\sigma_{r}=-70 \mathrm{MPa}$ is the initial residual stress in top coating [30]. The calculated thermal stress in ceramic coating is $155.9 \mathrm{MPa}$ at $T$ $=800^{\circ} \mathrm{C}$. After cooling to room temperature, thermal stress in top coating is equal to initial residual stress because of elastic recovery.

Obviously, stress in ceramic coat is tensile in the heating stage and reverses to compressive in the cooling stage. The tensile stress is detrimental to the coating lifetime because it tends to aid the opening and propagating of vertical cracks in ceramic coat. 
Although the compressive stress of ceramic coat tends to close vertical cracks and stops their propagation, it could promote the formation and propagation of interface cracks, which in turn lead to delamination between ceramic and bond coats and the subsequent spallation of ceramic coat. This is in well agreement with AE results, where preferential surface vertical cracks form during heating and interface cracks predominate in the period of cooling. Therefore, both the tensile stress in heating and compressive stress in cooling may result in damage in TBCs, which should not be neglected in predicting the lifetime of TBCs or in understanding micro-failure occurred in a full thermal cycled process.

\subsection{Surface crack density}

A shear lag model developed by McGuigan et al [24] is used to evaluate the surface crack density $\rho$, which is defined as the ratio of multiple crack numbers to the length of TBCs. This model involves a three-layer system consisting of a strained substrate, a relatively soft elastic-plastic interlayer of thickness $d_{2}$, and a thin brittle layer of thickness $d_{1}$, which agrees well with the structure of TBCs. When substrate is subjected to tensile strain, brittle coating splits vertically in the loading direction and the density of vertical cracks depends on whether plastic deformation occurs in substrate. If substrate is in a fully elastic state, the crack density $\rho$ of coating can be expressed as,

$$
\rho=\frac{k}{2} \operatorname{arccosh}\left(\frac{-G \varepsilon}{\sigma_{b} d_{1} d_{2} k^{2}-G \varepsilon}\right)^{-1}
$$

Otherwise, if substrate is in the elastic-plastic state, the crack density $\rho$ of coating can be expressed as,

$$
\rho=\frac{\tau_{b} k^{2} d_{2}}{2 G}(A+B+C)^{-1}
$$


where

$$
\begin{gathered}
A=\frac{\sigma_{b} k^{2} d_{1} d_{2}-G \varepsilon}{G} \cosh \left(\operatorname{arcsinh}\left(\frac{\tau_{b} k d_{2}}{G \varepsilon-\sigma_{b} k^{2} d_{1} d_{2}}\right)\right), \\
B=\frac{\tau_{b} k d_{2}}{G} \operatorname{arcsinh}\left(\frac{\tau_{b} k d_{2}}{G \varepsilon-\sigma_{b} k^{2} d_{1} d_{2}}\right) \\
C=\varepsilon
\end{gathered}
$$

In Eqs. (4) and (5), $k=\sqrt{\frac{G}{d_{1} d_{2} E}}, G$ is the shear modulus of interlayer, $E$ is Young's modulus of brittle coating, $d_{1}$ and $d_{2}$ are thickness of brittle coating and interlayer, respectively, $\sigma_{b}$ is the tensile strength of brittle coating, $\tau_{b}$ is the interface shear strength, and $\varepsilon$ is the tensile strain of substrate. Obviously, if the substrate strain $\varepsilon$ is estimated, the crack density can be determined by Eq. (4) or (5).

During heating, ceramic coating experiences a gradual increasing tensile stress and reaches its maximum value at furnace temperature. However, tensile stress is not uniform along the length direction of TBCs with an average value estimated by Eq. (2). Based on the shear-lag consideration, stress that ceramic coating experiences must be transferred from substrate to interface. In addition, a TBC system must satisfy the strain compatibility, and then the substrate strain $\varepsilon$ can be estimated by Eq. (3). For simplicity, fatigue is assumed as a simply linear superposition of damage in each cycle, which is suitable to an early fracture stage of TBCs. Thus, the cumulative surface damage can be calculated by the product of surface crack density $\rho$ and the number of cycles. Let the initial residual stress $\sigma_{r}=-70$ MPa [30], the calculated tensile strain in substrate is $0.29 \%$. That is, plastic deformation occurs in substrate under the tensile strain, and therefore, the crack density should be assessed by Eq. (5). For TBC specimens, the tensile strength $\sigma_{b}$ of ceramic coating and the interface shear strength $\tau_{b}$ were chosen in the range of 40 to $80 \mathrm{MPa}[33,34]$ and 6 to $20 \mathrm{MPa}[35,36]$, respectively. 
The relationships of calculated and experimental data for the surface vertical crack density and cumulative AE events with cycle numbers, and the correlation between the crack density and AE events are given in Figs. 8(a) and (b), respectively. It is obvious that the surface vertical crack density determined by the shear-lag model increases linearly with the increase of cycles due to a linear simplification of fatigue. In the case of $\sigma_{b}$ and $\tau_{b}$ being restricted to 80 and $12 \mathrm{MPa}$, experimental data are well consistent with the theoretical curve besides the late stage of cycles, as shown in Fig. 8(a). However, if the values of $\sigma_{b}$ and $\tau_{b}$ are expanded, all the experimental data, as shown in Fig. 8(b), locate between the range of calculated results with tensile and shear strengths are 80 and $12 \mathrm{MPa}$ or 60 and $12 \mathrm{MPa}$, respectively. These results indicate that the correlation between crack densities and $\mathrm{AE}$ activities determined by the shear-lag model is reliable and accurate to quantitatively assess the damage evolution in TBCs. Thus, the linear correlation between the crack density and AE events can be deduced. The calculated crack densities show the same tendency with a relative small slope as AE counts increase to about 120. It is also revealed in Fig. 8(b) that the weak interface layer, with a small value of $\tau_{b}$ and a high coating tensile strength $\sigma_{b}$, leads to a small crack density. As shown in Fig. 9, the initial residual stress plays no or little role on the crack density for various combinations of substrate yield strength and coating tensile strength, indicating that, for a given $\mathrm{TBC}$, its procedure process may have no influence on surface vertical cracks.

\subsection{Interface crack propagation}

As discussed above, the failure modes at the heating stage were mainly surface vertical cracks, and the delamination crack propagation was assessed only in the cooling stage. Under 
cycle loading, the crack propagation rate $\mathrm{d} a / \mathrm{d} N$ can be generally expressed as,

$$
\mathrm{d} a / \mathrm{d} N=C \Delta K^{m}
$$

where $\Delta K$ is the stress intensity factor amplitude associated with the cooling load, $C$ and $m$ are constants. Due to a little temperature difference across ceramic coating and a nearly uniform heating-cooling cycle, the residual compressive stress becomes a more predominant driving force for the interface crack propagation, primarily because of the elastic recovery of dynamic transient thermal stresses at cooling. Therefore, only the initial residual stress was considered here for the propagation of interface cracks.

To assess the effect of the stress intensity factor amplitude $\Delta K$ on interface crack propagation, a pure-shear interfacial mode was studied [25]. Based on the compressed stress amplitude, the driving force $\Delta K$ of subcritical crack propagation can be estimated by [25],

$$
\Delta K=F\left(\frac{a}{W}\right) \Delta \sigma \sqrt{\pi a}=\frac{a / W}{\sqrt{\pi(1-a / W)}}\left[7.264-9.37\left(\frac{a}{W}\right)+2.74\left(\frac{a}{W}\right)^{2}+\ldots\right] \Delta \sigma \sqrt{\pi a}
$$

where $\Delta \sigma$ is the stress amplitude calculated by the initial residual stress, $a$ is the interface crack length, $W$ is the depth of TBC specimens with $0 \leq \frac{a}{W} \leq 1$, and $F\left(\frac{a}{W}\right)$ is the crack geometry factor. For TBC specimens in this work, $W=3 \mathrm{~mm}$ and the crack length $a$ is in the range of micro-size. Thus, the value of $a / W$ is approximately equal to zero, and the crack geometry factor can be simplified as, $F(a / W)=7.264 \frac{a / W}{\sqrt{\pi}}$.

For the simplicity of calculation, let us introduce a nondimensional crack length $\bar{a}=a / a_{0}$ and the stress intensity factor $\overline{\Delta K}_{\mathrm{II}}=\frac{\Delta K}{K_{\mathrm{IIC}}}$ into Eq. (6). Substituting the simplified type of Eq. (7) into the dimensionless function of Eq. (6), the interface crack length can be expressed as, 


$$
a=a_{0}\left[\left(1-\frac{3}{2} m\right) D\left(\frac{7.264 a_{0}^{3 / 2} \Delta \sigma}{K_{\mathrm{IIC}} W}\right)^{m} N+1\right]^{\frac{2}{2-3 m}}
$$

where $a_{0}$ is the initial crack length, $\mathrm{D}$ is constant, $K_{I I C}$ is the interface fracture toughness, and $N$ is the cycle number.

In the calculation of interface crack propagation, the values of $a_{0}$ and $a$ at the 140th cycle are chosen as 1 and $18 \mu \mathrm{m}$, respectively, based on experimental observations as shown in Fig. 5. The exponent, $m$, for the delamination fatigue crack growth under the cyclic heating and cooling driving force $\Delta K$, was chosen in the range of 0 to 2 . The fracture toughness of TBCs was $0.73 \mathrm{MPa} \sqrt{\mathrm{m}}$ [25]. When $m$ and $\sigma_{r}$ were restricted to 0.6 and -70 MPa [30], respectively, the relationships of calculated and experimental data for the interface crack length with AE events are plotted in Fig. 10(a). Although there is some scatter of experimental data around the curve of calculated interface crack length, these data show a similar increasing law, indicating that the theoretical assessment is reliable. In addition, it is seen that, although $\mathrm{AE}$ events and interface length increase with the increase of cycles, there is no obvious linear or other tendency that could be established. Therefore, the correlations of interface crack lengths determined by both theoretical estimation and experimental observation with AE events are illustrated in Fig. 10(b), which allow us to quantify the interface crack damage evolution. The increasing tendency between AE signals and interface crack propagation can be divided into three regions: The first one starts with the beginning of tests and ends at the cycle with the total AE signals of about 70. As expected, the distribution of AE signals and their correlation with the interface crack length are irregular, based on the uncertainty in the early damage evolution. With the onset of macro-cracks, the count of AE events increases linearly with the interface crack propagation. 
Such a stable propagation process of interface cracks is defined as the second region, as shown in Fig. 10(b), with the range from 70 to 250 in the horizontal coordinate. In the last region, the unstable increment relationship of crack lengths and $\mathrm{AE}$ event count can be found out with a relative high slope attributed to the rapid propagation of delamination cracks at the late stage of testing. These results suggest that, for a given $\mathrm{TBC}$, if the delamination damage kinetics is concerned, its real-time monitoring of AE signals would give a linear correlation with the crack length.

It is obvious that, as shown in Fig. 10, the interface propagation behavior is clearly dependent on the values of $m$, where larger ones tend to advance the unstable growth with small crack lengths at the beginning of the third stage. In the case of $m$ being restricted to 0.6 , the relationships of interface crack length with $\mathrm{AE}$ signals and cycles under different initial residual stresses are plotted in Figs. 11(a) and (b), respectively. A crack did not grow without residual stress, which is reasonable because of no driving force for the crack propagation. A similar increasing tendency of the interface crack length with AE events was seen in Fig. 11(a), where larger values were corresponding to a larger residual stress, as shown in Fig. 11(b). For example, the interface crack length at the 140th cycle increases from 8.57 to $32.2 \mu \mathrm{m}$, which has a strong dependence on the initial residual stress from 40 to $100 \mathrm{MPa}$. This suggests that the control on residual stress induced by a deposition process can extend the service life of TBCs.

\section{Precursory time of coating spallation}

While micro-cracks are vital to the TBC performance, their propagation and coalescence are not catastrophic until the large-scale cleavage or spallation of coating occurs. Therefore, 
an important issue is how to predict the occurrence time of such a critical fracture, namely, a critical point or precursory time. In TBCs, single isolated microcracks firstly appear, and then, with the increase of load or time of loading, they grow and multiply, leading to an increase of the crack density or length $[3,5,6,37]$. As a consequence, microcracks begin to merge until a critical density (or a critical length) of cracks is reached, at which the main fracture occurs. The formation of micro-fractures prior to a major failure plays a crucial role in the fracture behavior, and naturally determines ultimate fracture and the service lifetime of TBCs. Moreover, these microcracks are correlated with each other in space and time [3, 6, 37]. Therefore, the criticality or precursory phenomena can be traced back by the interplay between the pre-existing heterogeneity and the correlated growth of cracks mediated by the singularities of damage parameters. Based on experimental observations discussed in Section 3, the AE response gives real-time and reliable information on the damage evolution in TBCs, indicating a qualitative physical picture for the progressive damage can be established through AE signals.

To study the precursory phenomena from recognizable signatures of microcracks, a statistical model was introduced according to the renormalization group theory in statistical physics $[38,39]$. The usefulness of renormalization is dependent on the existence of scale invariance at the critical point, which allows one to define a mapping between the physical scale and distance from the critical point in the control parameter axis [39]. Based on our recent work [19], the cumulative number distribution of $\mathrm{AE}$ events in TBCs follows a power-law or scaling, which implies that damage in TBCs gradually evolves to a critical point. Similar to AE events in other rupture phenomena [40, 41], a log-periodic correction to scaling can be used to fit the cumulative number or amplitude of AE signals in TBCs. Thus, the 
cumulative count or amplitude $f(N)$ of AE events versus the fracture evolution or the thermal cycle number $N$ in TBCs can be expressed as,

$$
f(N)=A+B\left(N_{f}-N\right)^{m}\left[1+C \cos \left(2 \pi \frac{\log \left(N_{f}-N\right)}{\log \lambda}+\varphi\right)\right]
$$

where $A, B$, and $C$ are normalizing parameters, $N_{f}$ is the critical cycle number to be predicted, $m$ is the exponent of the power-law growth, $\lambda$ is the scale ratio, and $\varphi$ is a shift parameter [41]. Here, it is worth noting that, relative to other formulae with a small number of fitting parameters, the amount of information obtained from this 7-parameter formula in Eq. (9) is significant $[40,41]$.

As shown in Fig. 12, Eq. (9) was used to fit the cumulative AE events and their corresponding cumulative amplitudes from TBCs in heating, cooling and whole cycling stages. It is seen that the fitting curves are in good agreement with experimental data in the heating stage for both AE event counts and amplitudes. The scatters of cooling and cycling data appear to fluctuate periodically as $N$ approaches $N_{f}$, indicating the reliability of prediction. The predicted values of precursory cycles are 221, 151, 164 and 203, 178, 150, for AE event counts and amplitudes, respectively, by fitting the data from heating, cooling, and whole cycling stages. The microstructures observed after 200 cycles reveal that a large scale of cleavage and delamination occurs in the ceramic coating and ceramic/bond interface, respectively. It is interesting to note that the predicted cycles through $\mathrm{AE}$ data from the heating stage are bigger than that in the cooling stage, moreover the latter ones exactly appear closing to those obtained from the whole cycling stage. It is also worth noting that an implied assumption here is that the precursory time is strongly dependent on the risk level of a failure type in TBCs. Under this assumption, for a given thermal cycle, all the possible failure types in TBCs may result in the critical damage when the released elastic energy reaches a state, i.e., 
the occurrence of singularity of AE signals. These results indicate that delamination damage at the ceramic/bond interface is more dangerous than cleavage damage in ceramic coating in determining the lifetime of TBCs.

\section{Conclusions}

In this paper, a systematic study has been carried out by using a real-time AE method on quantitative assessment of the fracture behavior in TBCs, subjected to cyclic heating and cooling. The following important conclusions can be made:

(1) Due to the excellent response of time- and frequency-domains of AE signals, wavelet transform is proved to be a useful way to discriminate failure modes in TBCs.

(2) Coating failures can occur in both heating and cooling stages during thermal cycling. The results obtained by the wavelet analysis of AE signals reveal that damage exhibits mainly surface vertical cracking in the heating stage and interface cracking in the cooling stage. The failure mechanism can be explained based on the stress state in TBCs, which experiences a tensile stress in the heating stage and then turns to a compressive stress in the cooling stage, leading to the surface vertical cracking and interface cracking, respectively.

(3) The quantitative parameters, such as the surface crack density and interface crack length, estimated by theoretical models have a well correlated relationship with AE events associated with each failure mode. It provides a potential possibility for the quantitative assessment of damage evolution in TBCs.

(4) The predicted precursory time is well agreement with the experimental observation of large scale cleavage and delamination. In the whole failure controlled by a singularity of AE signals, delamination damage is more dangerous than cleavage damage for the lifetime of 
TBCs.

These results have shown that $\mathrm{AE}$ can be a powerful and reliable tool for assessing the fracture behavior in TBCs and predicting their service life. It is worth noting that, however, our analysis, including the quantitative assessment of damage evolution and precursory time prediction of TBCs, is still at a very primitive stage. More studies will be needed on the quantitative assessment of damage evolution in TBCs to obtain an equation describing the relationship between mirocracks and AE activities.

\section{Acknowledgements}

This research has been supported by the National Natural Science Foundation of China (Nos. 11002122, 50531060, 11002121, and 10828205), the Natural Science Foundation of Hunan Province for Innovation Group (No. 09JJ7004), and the Open Project of State Key Laboratory of Explosion Science and Technology of China (No. KFJJ07-7). 


\section{References}

[1] Padture NP, Gell M, Jordan EH. Science 2002;296:280.

[2] Miller RA. Surf Coat Technol 1987;1:30.

[3] Evans AG, Mumm DR, Hutchinson JW, Merier GH, Petit FS. Prog Mater Sci 2001;46:505.

[4] Chang GC, Phucharoen W, Miller RA. Surf Coat Technol 1987;30:13.

[5] Chen X, Hutchinson JW, He MY, Evans AG. Acta Mater 2003;51:2017.

[6] Busso EP, Wright L, Evans HE, McCartney LN, Saunders SRJ, Osgerby S, Nunn J. Acta Mater 2007;55:1491.

[7] Balint DS, Hutchinson JW. J Mech Phys Solids 2005;53:949.

[8] Hille TS, Nijdam TJ, Suiker AJ, Turteltaub S, Sloof WG. Acta Mater 2009; 57:2624

[9] Veal BW, Paulikas AP, Hou PY. Nat Mater 2006;5:349.

[10] Zhang CX, Zhou CG, Gong SK, Li HF, Xu HB. Surf Coat Tech 2005;13:315.

[11] Yu FL, Bennett TD. J Appl Phys 2005;98:103501.

[12] Christensen RJ, Lipkin DM, Clarke DR. Appl Phys Lett 1996;69:3754.

[13] Thornton J, Cookson D, Pescott E. Surf Coat Tech 1999;120-121:96.

[14] Renusch D, Schütze M. Surf Coat Technol 2007;202:740.

[15] Ma XQ, Takemoto M. Mater Sci Eng A 2001;38:101.

[16] Fu L, Khor KA, Ng HW, Teo TN. Surf Coat Technol 2000;130:233.

[17] Ma XQ, Cho S, Takemoto M. Surf Coat Technol 2001;139:55.

[18] Yang L, Zhou YC, Mao WG, Liu QX. Surf Interface Anal 2007;39:761.

[19] Yang L, Zhou YC, Mao WG, Lu C. Appl Phys Lett 2008;93:231906.

[20] Benson PM, Vinciguerra S, Meredith PG, Young RP. Science 2008;322:248. 
[21] Chui CK. An Introduction to Wavelets. San Diego, CA: Academic Press, 1992.

[22] Newland DE, Random Vibrations, Spectral and Wavelet Analysis. Essex, UK: Longman Scientific \& Technical, 1993.

[23] Daubechies I. IEEE T Inform Theory 1990;36:961.

[24] McGuigan AP, Briggs GAD, Burlakov VM, Yanaka M, Tsukahara Y. Thin Solid films $2003 ; 424: 219$.

[25] Choi SR, Zhu D, Miller RA. Eng Fract Mech 2005;72:2144.

[26] Seong SH, Hur S, Kim JS, Kim JT, Park WM, Lee UC, Lee SK. Ann Nucl Energy 2005; $32: 479$.

[27] Mao WG, Chen Q, Dai CY, Yang L, Zhou YC, Lu C. Surf Coat Technol 2010;204:3573.

[28] Teixeira V, Andritschky M, Fischer W, Buchkremer HP, Stöver D. Surf Coat Technol 1999;120-121:103.

[29] Suzuki K, Shobu T. Mater Sci Forum 2010;638-642:906.

[30] Vasinonta A, Beuth JL. Eng Fract Mech 2001;68:843.

[31] Zhang XC, Xu BS, Wang HD, Wu YX. J Appl Phys 2007;101:083530.

[32] Hsueh CH. Thin Solid Films 2002;418:182.

[33] Zhou YC, Tonomori T, Yoshida A, Liu L, Bignall G, Hashida T. Surf Coat Technol $2002 ; 157: 118$.

[34] Schwingel D, Taylor R, Haubold T, Wigren J, Gualco C. Surf Coat Technol 1998;108-109:99.

[35] Xu ZH, Yang YC, Huang P, Li XD. Acta Mater 2010;58:5972.

[36] Cruse TA, Dommarco RC, Bastias PC. J Eng Mater Technol 1998;120:26.

[37] Courcier C, Maurel V, Rémy L, Quilici, Rouzou I. Phelippeau A. Surf Coat Technol 
2011;205:3763

[38] Wilson KG, Kogut J. Phys Rep 1974;12:75.

[39] Stanley HE. Rev Mod Phys 1999;71:S358.

[40] Bufe DG, Varnes DJ. J Geophys Res 1993;98:9871.

[41] Sornette D, Sammis CG. J Phy I France 1995;5:607. 


\section{Figure captions}

Fig. 1. Illustration of the research process on the damage evolution and rupture time prediction in TBCs by an AE method: (i) to monitor surface vertical or interface cracks and their corresponding AE signals, and then (ii) to correlate AE parameters with cracking information, including the crack type, crack density or length, and critical rupture time.

Fig. 2. Schematic of the experimental setup for cyclic heating and cooling tests and AE measurements.

Fig. 3. Typical distribution histograms of energy coefficients for surface vertical crack and interface crack based on wavelet transformation of AE signals.

Fig. 4. AE events monitored from TBCs under cyclic heating and cooling.

Fig. 5. Scanning electron microscopy patterns of TBCs for sample A after (a) 20, (b) 60, (c) 100, and (d) 140 cycles, and for sample B after (e) 20, (f) 60, (g) 100, and (h) 140 cycles at $800^{\circ} \mathrm{C}$, respectively.

Fig. 6. Relationships between (a) AE events and (b) their corresponding amplitude distributions with the cycle number during heating and cooling stages.

Fig. 7. The failure mode differentiation results of AE signals monitored in (a) heating, and (b) cooling stages, respectively.

Fig. 8. (a) Relationships of surface vertical crack density and cumulative AE events with the cycle number, and (b) the correlation between the crack density and AE events.

Fig. 9. The effect of initial residual stress on the crack density at different shear strengths of 
substrate and tensile strengths of coating.

Fig. 10. (a) Relationships of interface crack length and AE events with the cycle number, and (b) the correlation between the interface crack and AE events.

Fig. 11. The effect of initial residual stress on interface crack in TBCs subjected to cyclic heating and cooling. (a) The relationship of interface crack length with AE events, and (b) the relationship of interface crack length with the cycle number under different initial residual stresses.

Fig. 12. The fitting results of (a) cumulative $\mathrm{AE}$ events and (b) their corresponding cumulative amplitudes from TBCs in heating, cooling and whole cycling stages, respectively. 

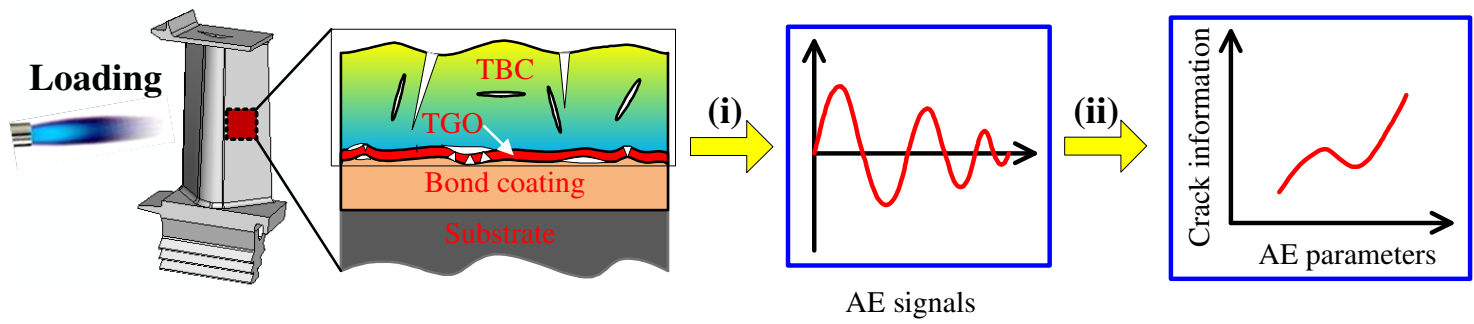

Fig. 1 


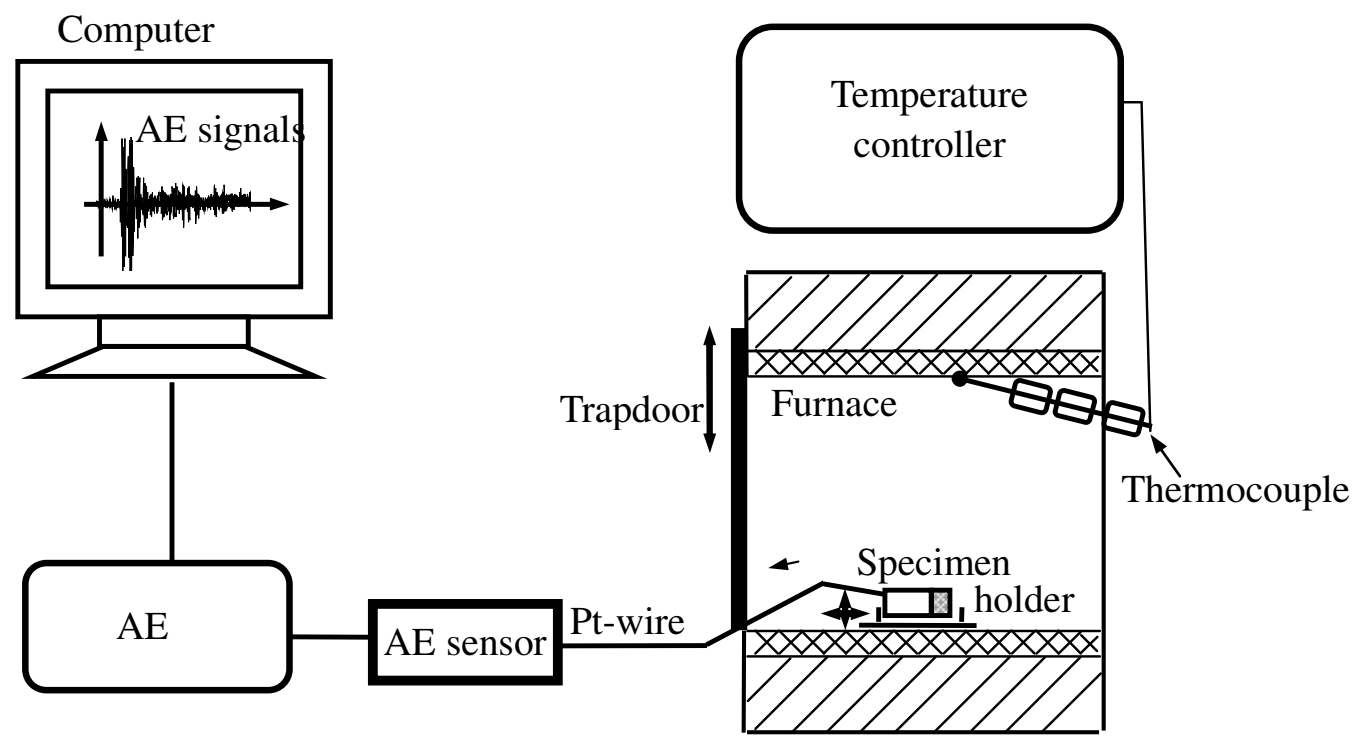

Fig. 2 


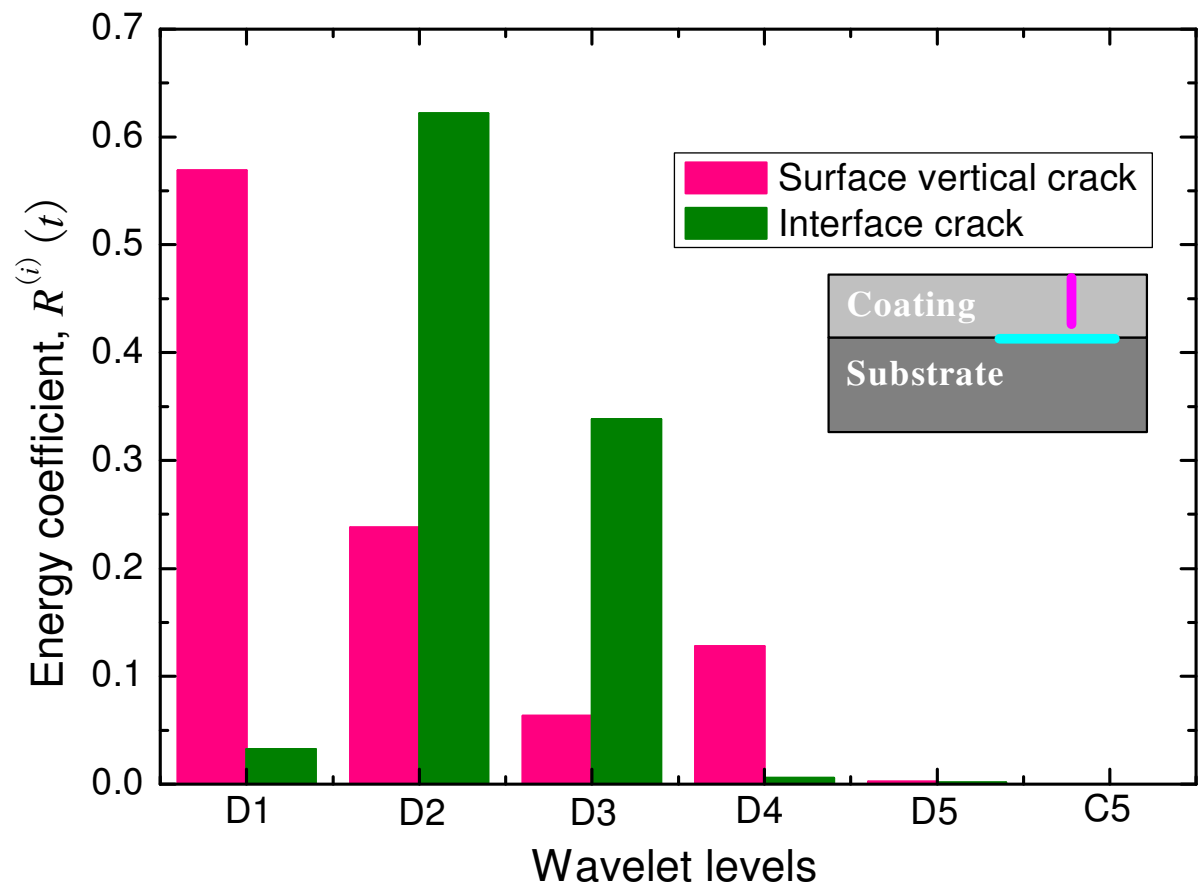

Fig. 3 


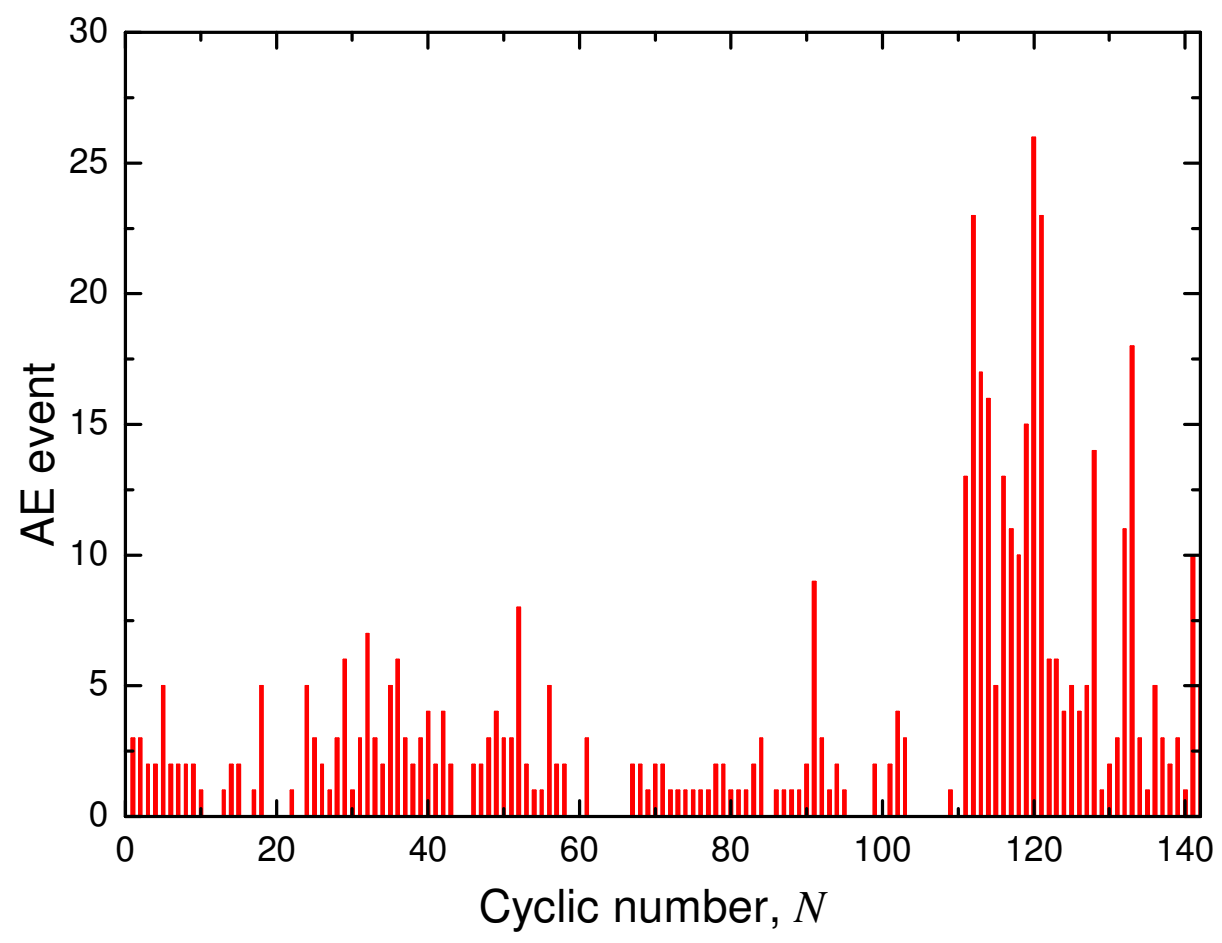

Fig. 4 

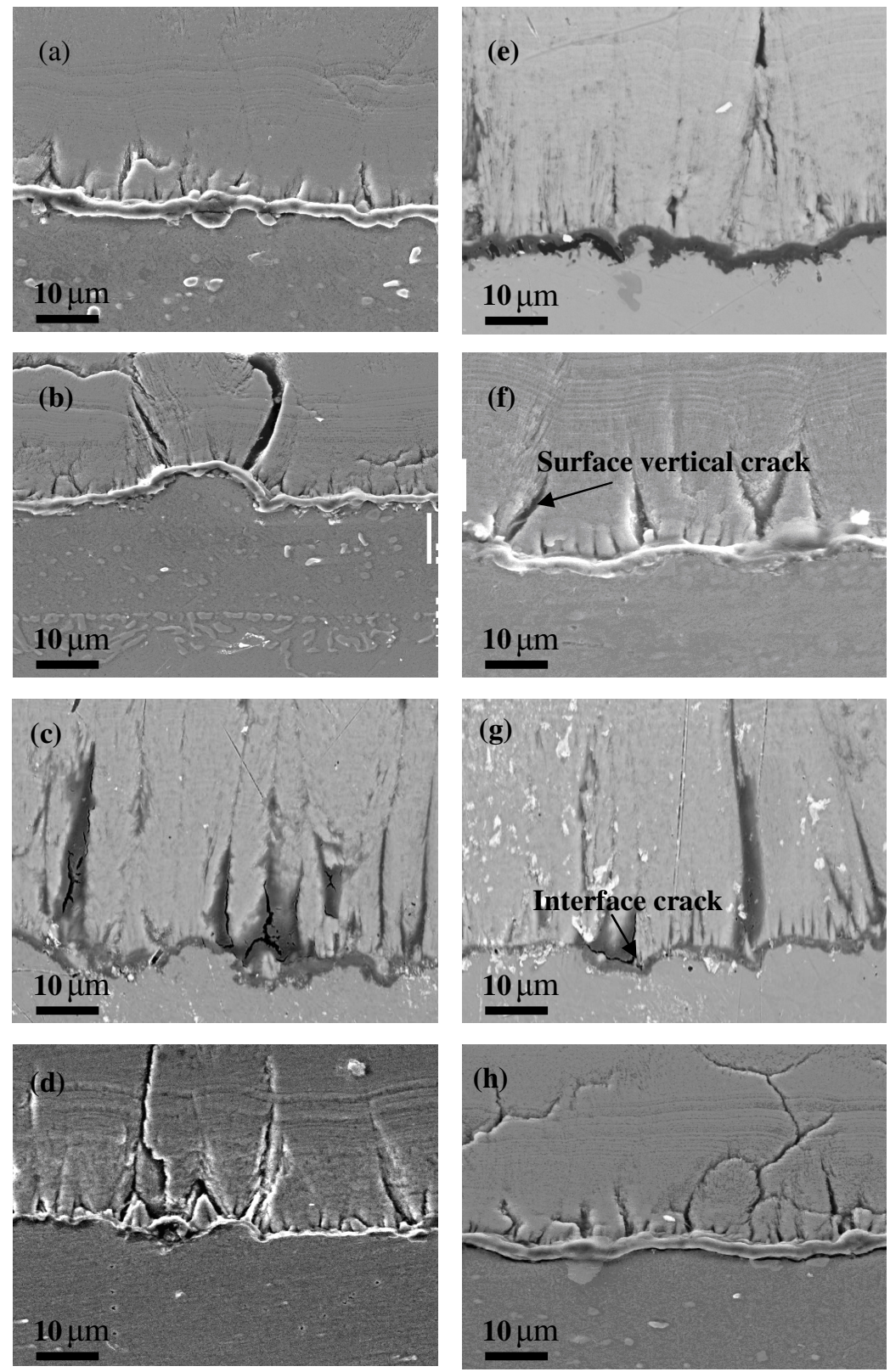

Fig. 5 

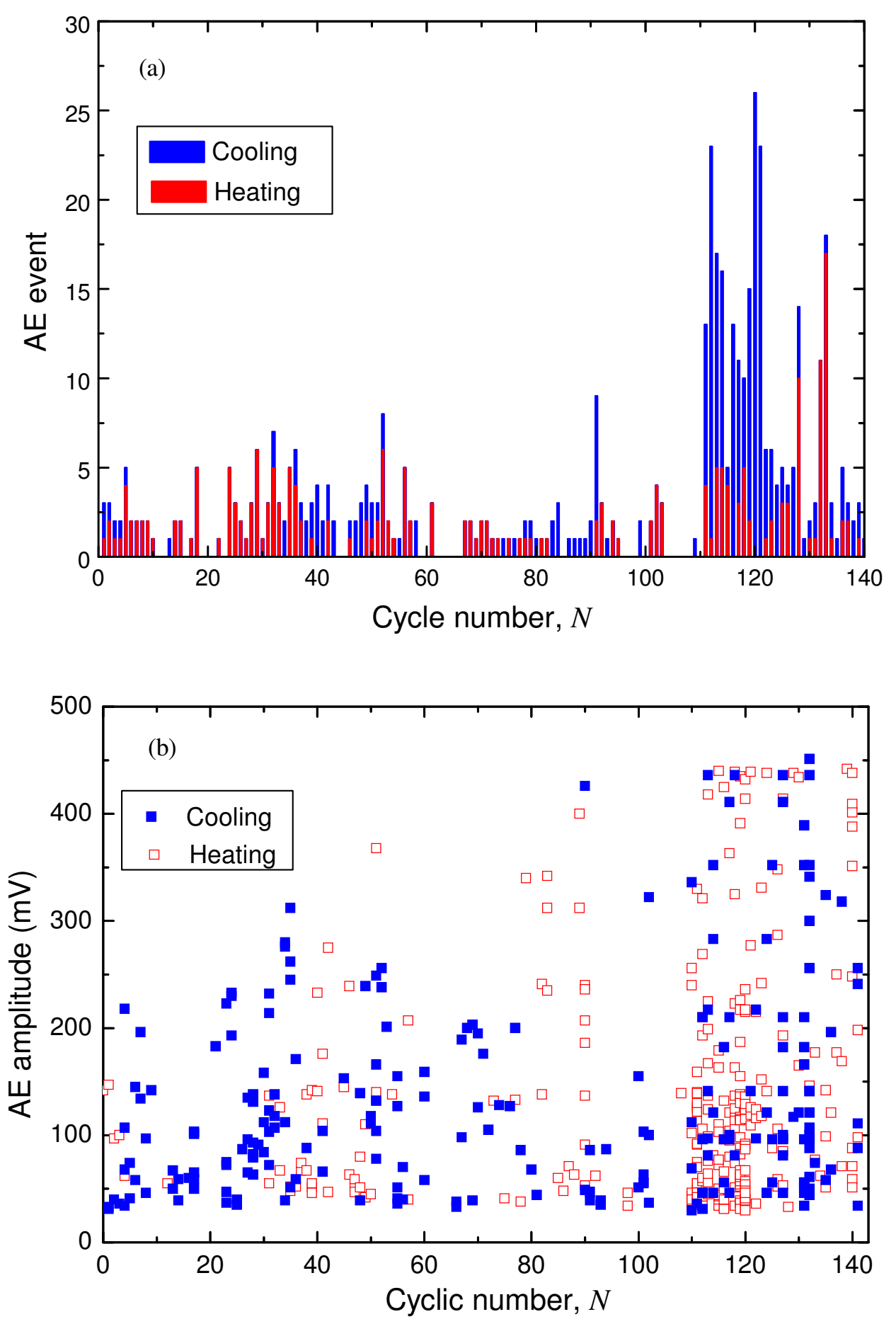

Fig. 6 

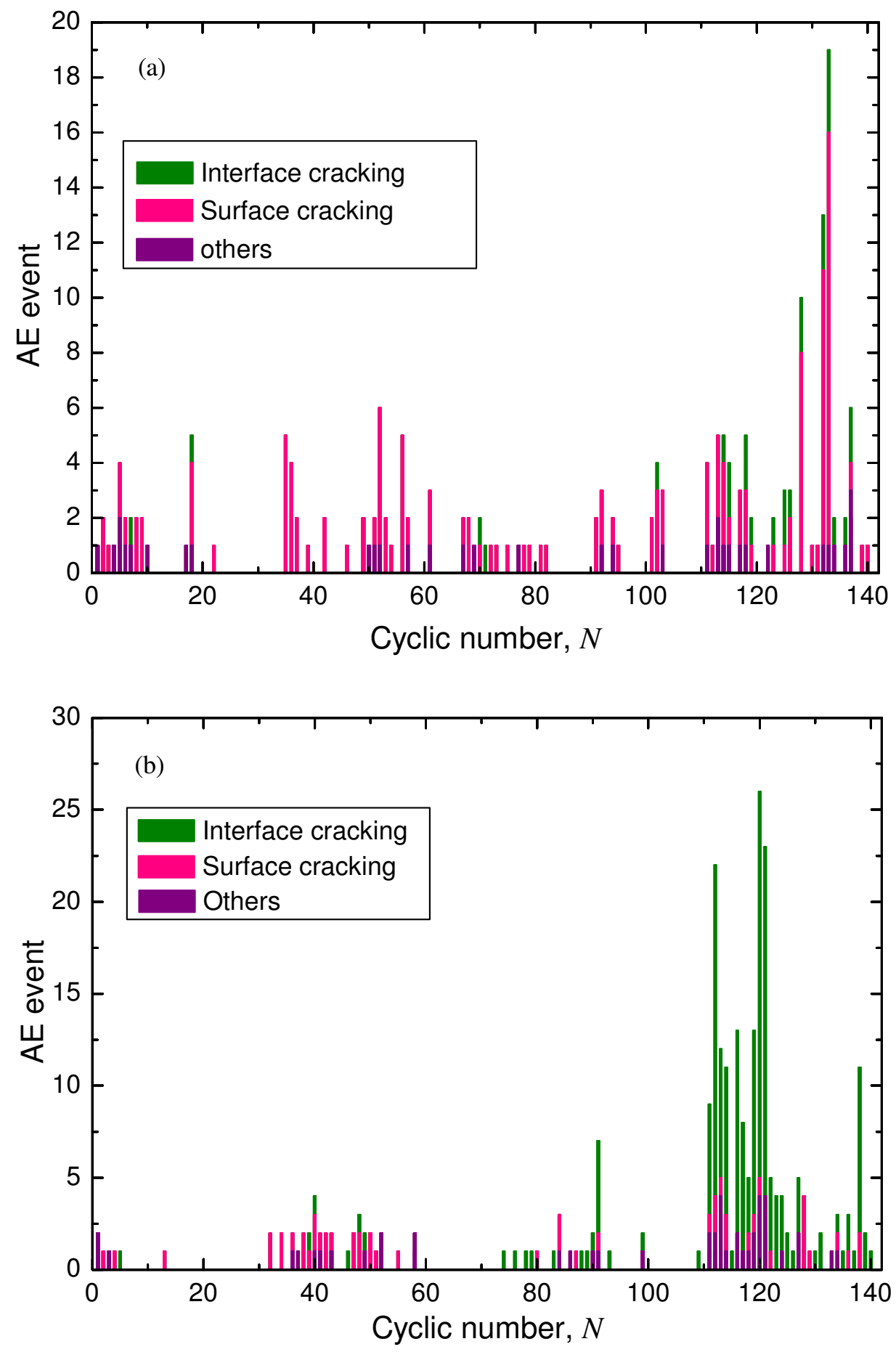

Fig. 7 

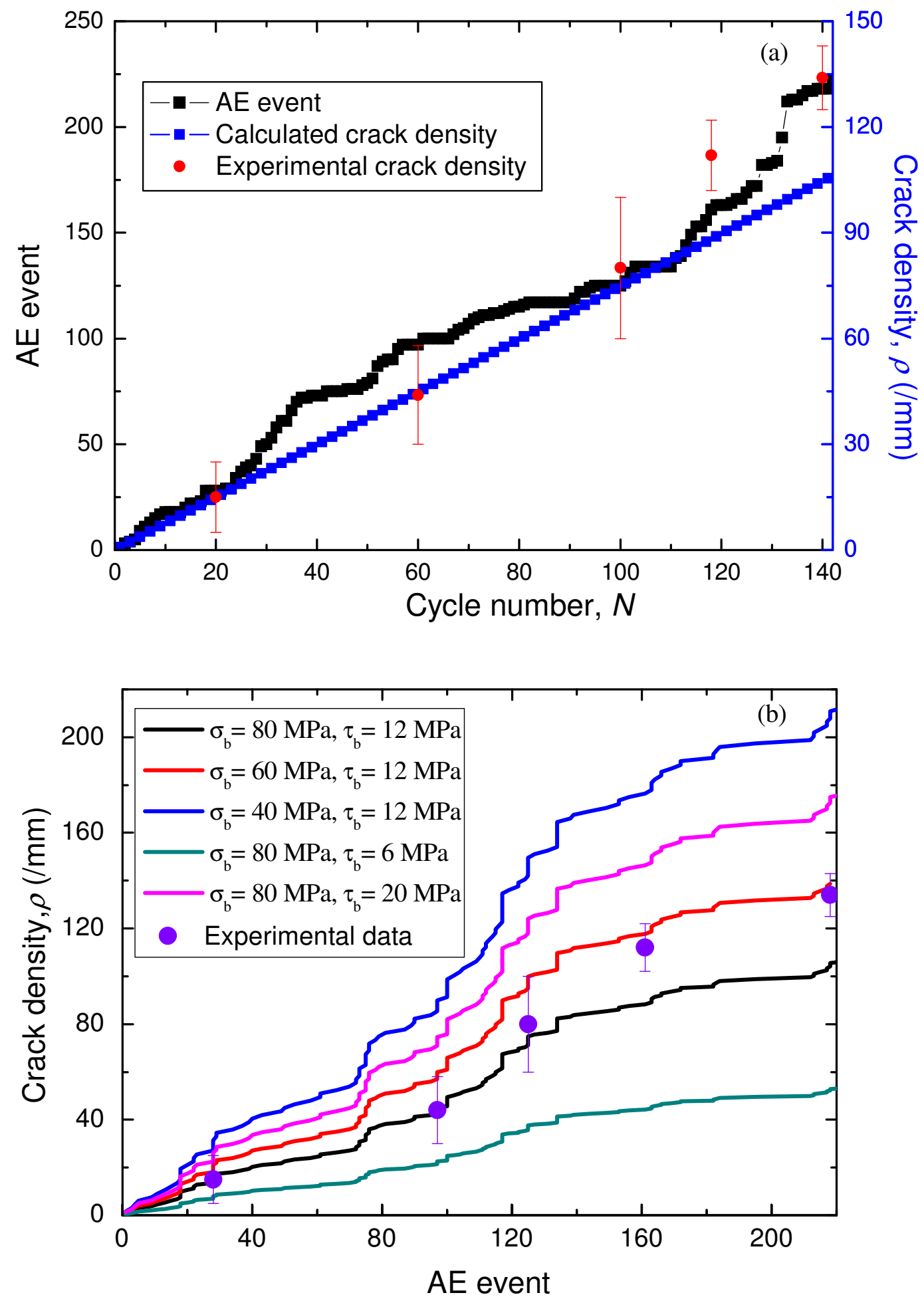

Fig. 8 


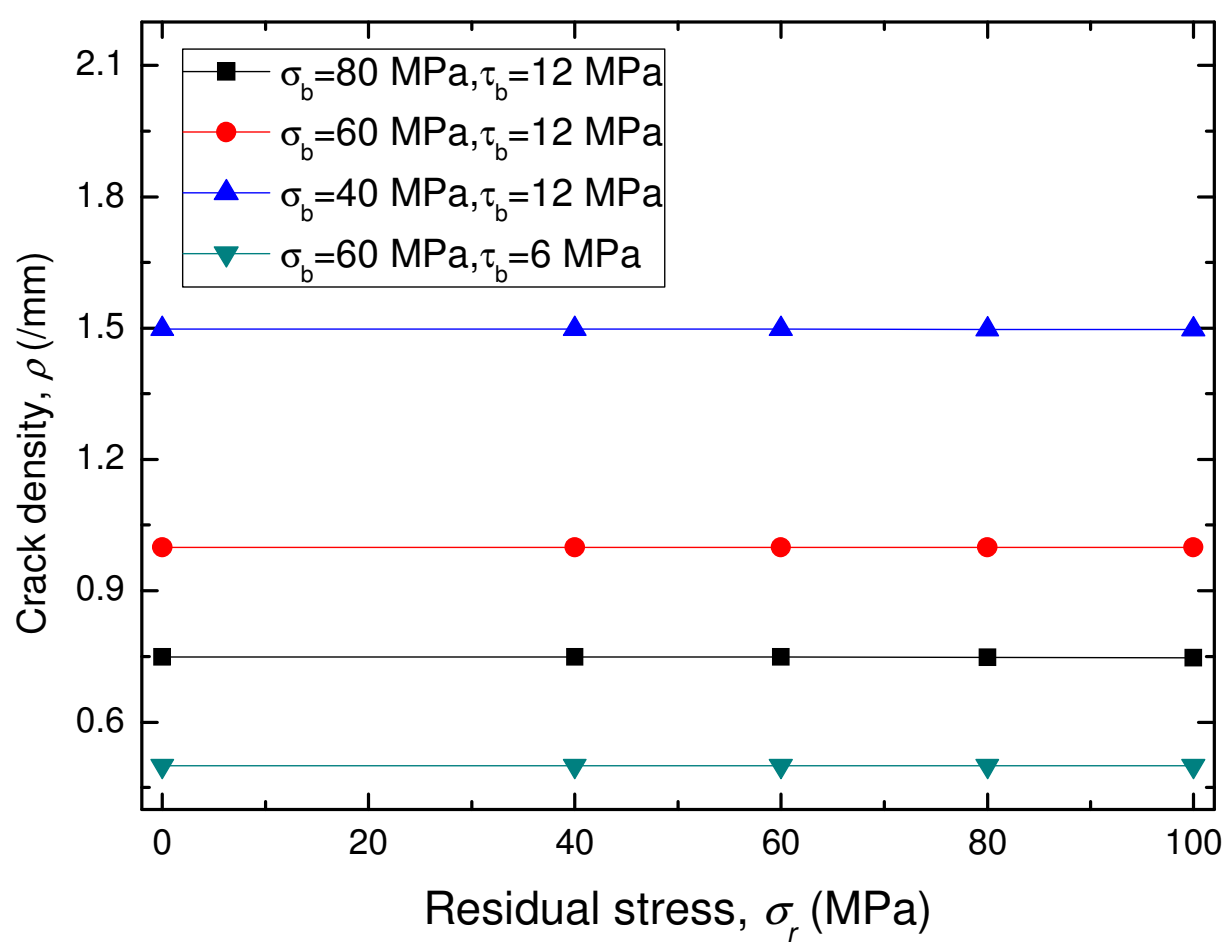

Fig. 9 

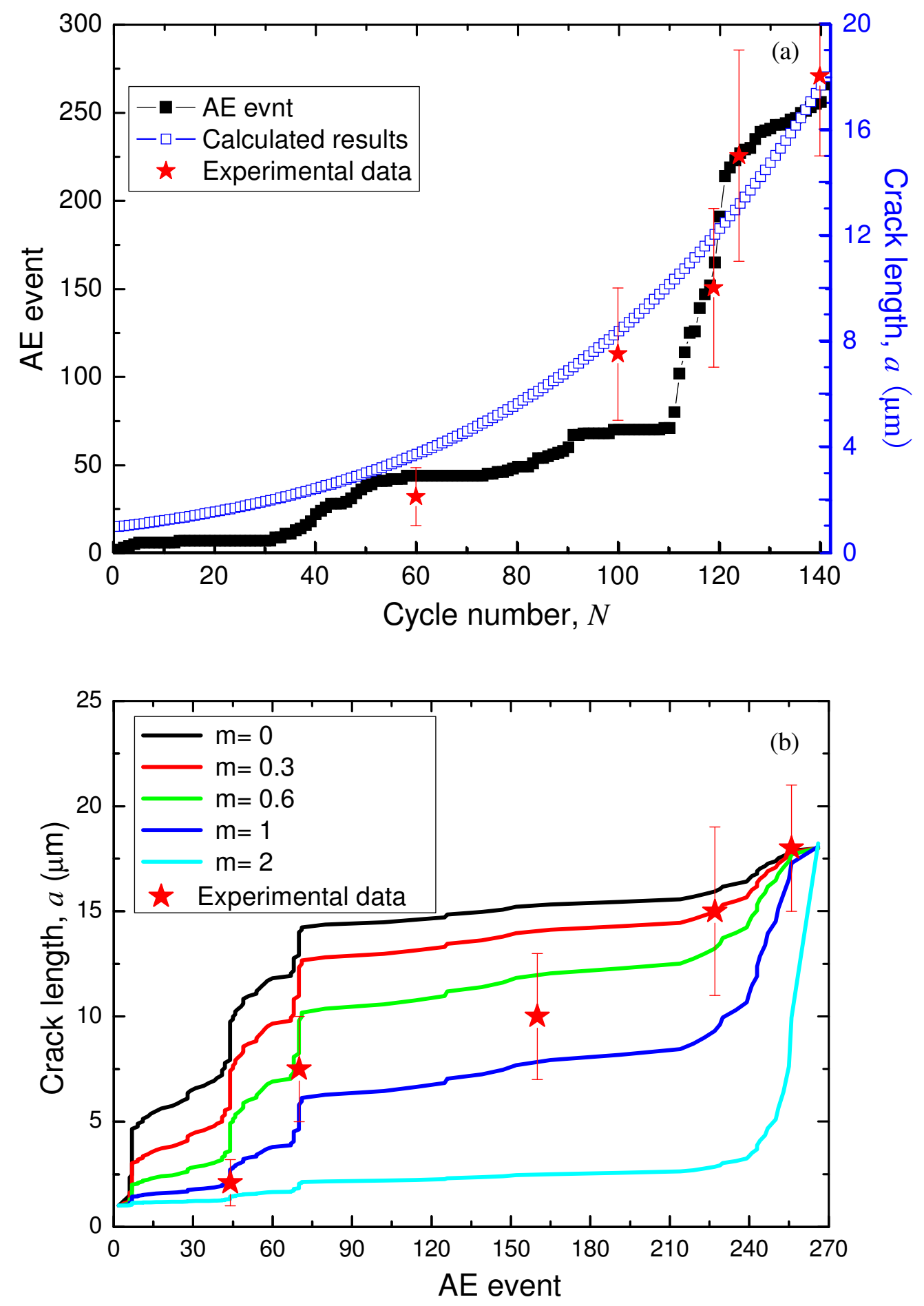

Fig. 10 

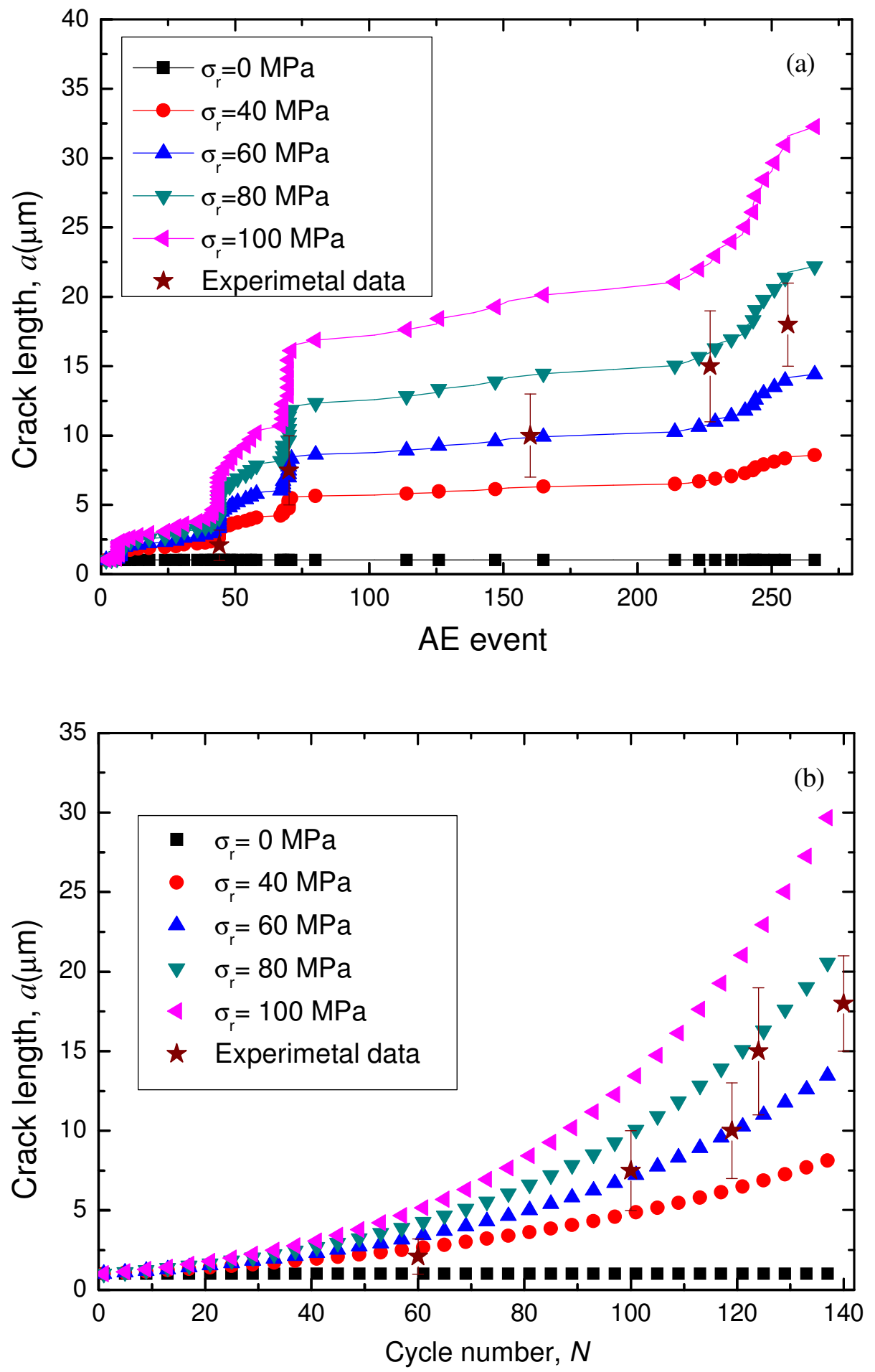

Fig. 11 

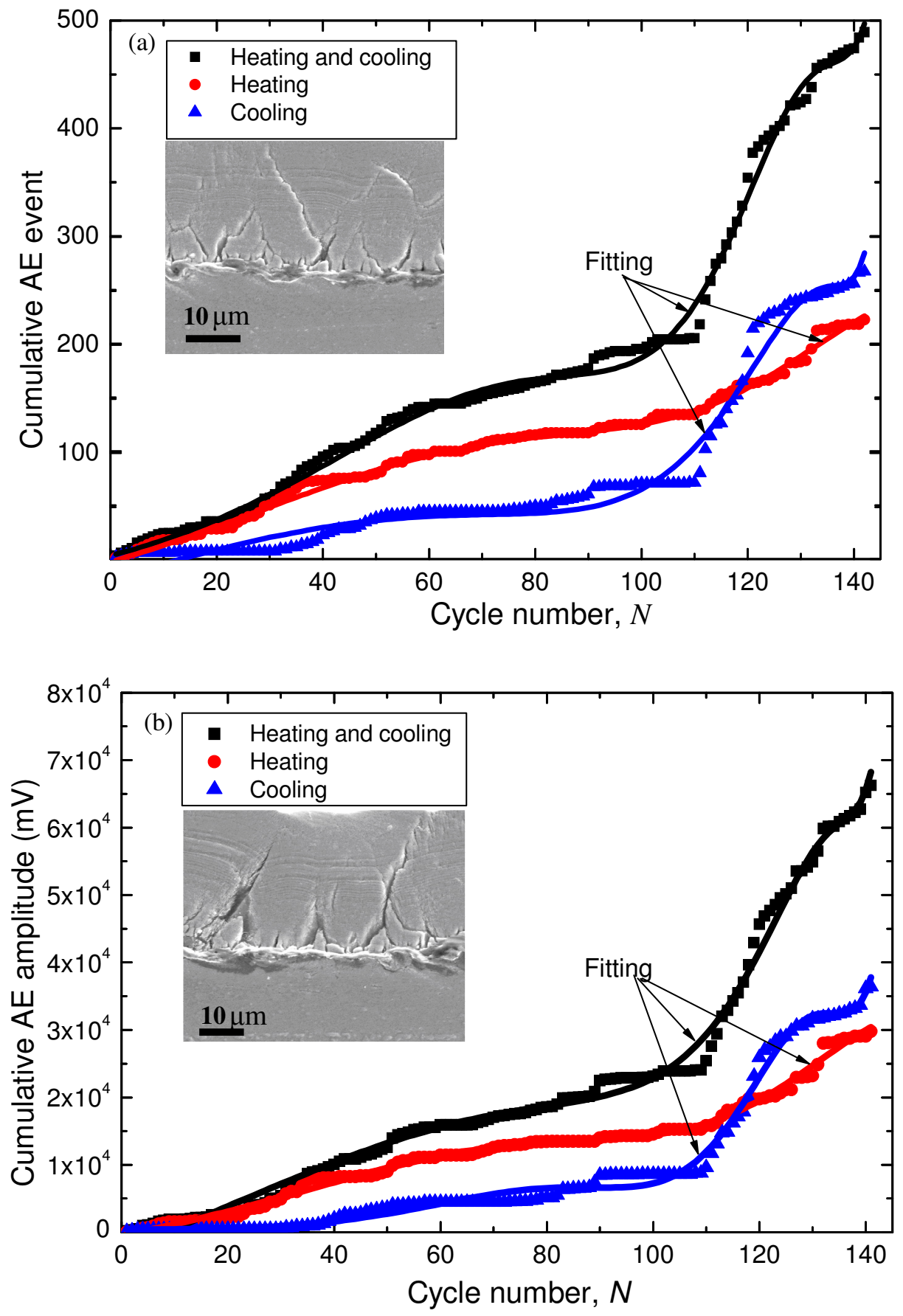

Fig. 12 